\title{
Interaction study between vancomycin and liposomes containing natural compounds against methicillin-resistant Staphylococcus aureus clinical isolates
}

\author{
Isabella Macário Ferro Cavalcanti ${ }^{1,2 *}$, Talita Gomes Calaça Menezes ${ }^{2}$, Luís André de Almeida \\ Campos $^{1,2}$, Milena Sales Ferraz ${ }^{2}$, Maria Amélia Vieira Maciel ${ }^{3}$, Maria Nelly Psiotano Caetano ${ }^{4}$, \\ Nereide Stela Santos-Magalhães ${ }^{2,4}$
}

\begin{abstract}
${ }^{1}$ Microbiology and Immunology Laboratory, Academic Center of Vitoria, Federal University of Pernambuco, Vitoria de Santo Antão, Pernambuco, Brazil, ${ }^{2}$ Immunopathology Laboratory Keizo Asami, Federal University of Pernambuco, Recife, Pernambuco, Brazil, ${ }^{3}$ Department of Tropical Medicine, Federal University of Pernambuco, Recife, Pernambuco, Brazil, ${ }^{4}$ Microbiological Analysis Laboratory, Department of Pharmaceutical Sciences, Federal University of Pernambuco, Recife, Pernambuco, Brazil
\end{abstract}

\begin{abstract}
The treatment of infections caused by resistant microorganisms is limited, and vancomycin (VAN) treatment failures for methicillin-resistant Staphylococcus aureus (MRSA) bacteremia are not uncommon, even when MRSA clinical isolates are susceptible to VAN. Thus, this study proposed the association of VAN with usnic acid and $\beta$-lapachone encapsulated into liposomes as a novel therapeutic option for infections caused by MRSA. Liposomes containing $\beta$-lap ( $\beta$-lap-lipo) or usnic acid (UA-lipo) were prepared by the thin lipid film hydration method followed by sonication. Antimicrobial activity against MRSA clinical isolates was investigated by the microdilution method according to the Clinical and Laboratory Standards Institute (CLSI). The interaction studies were carried out using the checkerboard method and epsilometer test (Etest). The interaction between VAN and $\beta$-lap or $\beta$-lap-lipo was synergistic $($ FICI $=0.453$ and FICI $=0.358$, respectively). An additive interaction between VAN and UA $(\mathrm{FICI}=0.515)$ was found. UA-lipo resulted in synergism with VAN $(\mathrm{FICI}=0.276)$. The Etest reproduced the results obtained by the checkerboard method for approximately $82 \%$ of the analysis. Thus, the present study demonstrated that VAN in combination with UA-lipo, $\beta$-lap or $\beta$-lap-lipo synergistically enhanced antibacterial activity against MRSA.
\end{abstract}

Keywords: $\beta$-lapachone. Usnic acid. Liposomes. Methicillin-resistant Staphylococcus aureus (MRSA). Synergism.

\section{INTRODUCTION}

The emergence worldwide of multiresistant bacteria has limited conventional antimicrobial therapy and raises serious concerns for public health care systems. From this perspective, methicillin-resistant Staphylococcus aureus (MRSA) is highly virulent and infections caused by MRSA may lead to morbidity and mortality (Zecconi, Scali, 2013). Consequently, the scientific community

\footnotetext{
*Correspondence: Isabella Macário Ferro Cavalcanti. Laboratório de Imunopatologia Keizo-Asami (LIKA), Universidade Federal de Pernambuco. Av. Prof. Moraes Rego, 1235 - Cidade Universitária, 50670-901, Recife-PE, Brasil. Telefone: + 5581 21268587; Fax: +55 81 21268485. E-mail: bel_macario@ yahoo.com.br
}

is engaged in the development of novel antimicrobial agents, including combination therapy of antimicrobials and/or encapsulated molecules in drug delivery systems (Pinto-Alphandary, Andremont, Couvreur, 2000; Segatore et al., 2012).

In this scenario, the association of antimicrobial agents for the treatment of infections caused by multiresistant bacteria seems to be significant, since combined therapy may prevent the emergence of resistant isolates and also increase the spectrum of action of antimicrobials due to a synergic effect (Jackson, Agboke, Nwoke, 2009; Silva, Mesquita, Ximenes, 2009; Worthington, Melander, 2013). Vancomycin (VAN) is the reference drug for the treatment of MRSA; however, MRSA strains have presented 
vancomycin resistance in vivo even when the in vitro profile was intermediate or susceptible for this drug (Moise et al., 2007; Casapao et al., 2015).

Furthermore, new compounds of natural origin that exhibit antimicrobial activity have been discovered. Therefore, usnic acid (UA), a secondary lichen metabolite, and $\beta$-lapachone ( $\beta$-lap), a naphthoquinone derived from lapachol extracted from Tabebuia avellanedae bark have been studied to establish their antimicrobial activity (Lauterwein et al., 1995; Lira et al., 2009; Macedo et al., 2013). Although these molecules exhibit proven antimicrobial activity, they have limitations, such as low water solubility and toxicity, which need to be overcome (Kristmundsdoóttir et al., 2002; Nasongkla et al., 2003; Han et al., 2004).

Nanostructured systems, such as liposomes, have great appeal as a means of delivery of antibiotics to treat infections. The encapsulation of antibiotics into liposomes offers several advantages, including improvement in their therapeutic efficacy and reduction in toxicity (DrulisKawa, Dorotkiewicz-Jach, 2010; Huh, Kwon, 2011). Moreover, nanocarriers containing antibiotics can deliver drugs to pathogenic bacteria, while avoiding killing commensal bacteria of the body, regulating the therapy of infections (Abed, Couvreur, 2014).

Taking into account all these findings, the combination of vancomycin with potential compounds of natural origin alone or encapsulated into nanosystems represents an option to improve the therapeutic index of vancomycin and overcome resistance to this antimicrobial agent. Thus, the aim of this study was to evaluate the in vitro interactions between vancomycin and usnic acid or $\beta$-lapachone encapsulated into liposomes against methicillin-resistant Staphylococcus aureus (MRSA) clinical isolates.

\section{MATERIAL AND METHODS}

\section{Material}

Vancomycin hydrochloride (VAN) was furnished by ABL - Antibióticos do Brasil (Cosmópolis, São Paulo, Brazil). Usnic acid (UA), cholesterol (Chol) and stearylamine (SA) were purchased from Sigma-Aldrich (St. Louis, USA). Soybean phosphatidylcholine (PC) (98\% Epikuron $200^{\circledR}$ ) was obtained from Lipoid GMBH (Ludwigshafen, Germany). Solvents and other chemicals were supplied by Merck (Darmstadt, Germany). Etest strips with vancomycin were purchased from Oxoid (Hampshire, United Kingdom). $\beta$-lapachone ( $\beta$-lap), obtained from lapachol by a semi-synthetic route, was supplied by Dr. Góes (Department of Antibiotics, UFPE, Brazil). Bacterial culture media were purchased from HiMedia (Mumbai, India).

\section{Methods}

\section{Preparation of liposomes}

Liposomes containing $\beta$-lapachone ( $\beta$-lap-lipo) or usnic acid (UA-lipo) were prepared according to Cavalcanti et al. (2011) and Lira et al. (2009), respectively, for comparative purposes in the antimicrobial activity evaluation and for the interaction assays with VAN. The liposomal formulations were prepared using the thin lipid film method followed by sonication. Briefly, lipids (PC, Chol and SA) and drugs ( $\beta$-lap or UA) were dissolved in a mixture of $\mathrm{CHCl}_{3}: \mathrm{CH}_{3} \mathrm{OH}(3: 1, \mathrm{v} / \mathrm{v})$ under magnetic stirring. The solvents were then removed under pressure for $60 \mathrm{~min}\left(37 \pm 1^{\circ} \mathrm{C}, 80 \mathrm{rpm}\right)$, resulting in a thin lipid film. This film was then hydrated with phosphate buffer solution (pH 7.4). Multilamellar liposomes were then sonicated (Vibra Cell; Branson Ultrasonic, Danbury, CT) at $200 \mathrm{~W}$ and $40 \mathrm{~Hz}$ for $300 \mathrm{~s}$ to form small unilamellar vesicles. The liposomes were characterized by measuring $\mathrm{pH}$, particle size, polydispersion index (PDI), zeta potential and encapsulation efficiency (\%EE), as previously described by Cavalcanti et al. (2011) and Lira et al. (2009).

\section{In vitro antimicrobial activity}

\section{Bacterial strains}

Staphylococcus aureus clinical isolates (10 strains) were furnished by the hospital of the Federal University of Pernambuco (UFPE, Recife, Brazil) and preserved in the Microbiology and Immunology Laboratory (CAVUFPE, Brazil). These strains were previously identified as MRSA using the disk diffusion method with cefoxitin and oxacillin as reference drugs, as well as by screening using Müeller-Hinton agar supplemented with $4 \% \mathrm{NaCl}$ and $6 \mu \mathrm{g} / \mathrm{mL}$ of oxacillin according to the Clinical and Laboratory Standards Institute (CLSI, 2014) (LMB 01, LMB 02, LMB 03, LMB 04, LMB 06, LMB 07, LMB 08, LMB 11, LMB 12 and LMB 13). MRSAATCC 33591 was used as the control.

\section{Determination of Minimum Inhibitory Concentration (MIC) and Minimum Bactericidal Concentration (MBC)}

The in vitro antimicrobial activity of VAN, UA and UA-lipo was evaluated by the microdilution method 
according to CLSI (2014). The antimicrobial activity of $\beta$-lap and $\beta$-lap-lipo against these MRSA clinical isolates has been previously reported by our research group (Cavalcanti et al., 2015). However their interactions with vancomycin have not yet been investigated.

\section{Interaction assays}

\section{Checkerboard method}

The in vitro interactions between VAN and UA, UA-lipo, $\beta$-lap or $\beta$-lap-lipo were evaluated using the checkerboard method (An et al., 2011). After the determination of MIC, the five MRSA clinical isolates most resistant to VAN were selected for this analysis. Initially, the 96-well microplates were seeded by dispersing Müeller Hinton broth into each well. Subsequently, serially diluted VAN was dispensed on the $\mathrm{X}$-axis of the 96-well microdilution plates and the test drugs (UA, UA-lipo, $\beta$-lap or $\beta$-lap-lipo) on the Y-axis, to obtain a final concentration equal to the MIC or dilutions lower than the MIC of the respective drugs. Finally, each plate received the adjusted bacterial suspension $\left(10^{5} \mathrm{CFU} /\right.$ $\mathrm{mL}$ ) and the plates were then incubated at $35^{\circ} \mathrm{C}$ for $24 \mathrm{~h}$.

The data were interpreted after calculating the Fractional Inhibitory Concentration Index (FICI) as follows:

$$
\Sigma \mathrm{FICI}=\mathrm{FIC}_{\mathrm{A}}+\mathrm{FIC}_{\mathrm{B}}=\mathrm{MIC}_{\mathrm{AB}} / \mathrm{MIC}_{\mathrm{A}}+\mathrm{MIC}_{\mathrm{BA}} / \mathrm{MIC}_{\mathrm{B}}
$$

where $\mathrm{MIC}_{\mathrm{AB}}$ equals the MIC of drug $\mathrm{A}$ in combination with drug $\mathrm{B}, \mathrm{MIC}_{\mathrm{A}}$ is the $\mathrm{MIC}$ of drug $\mathrm{A}$ alone, $\mathrm{MIC}_{\mathrm{BA}}$ equals the MIC of drug $B$ in combination with drug $A$ and $\mathrm{MIC}_{\mathrm{B}}$ is the MIC of drug $\mathrm{B}$ alone. The interaction is considered synergic for $\mathrm{FICI} \leq 0.5$; additive for $0.5<\mathrm{FICI} \leq 1$, indifferent for $1<\mathrm{FICI} \leq 2$ and antagonistic for FICI $>2$ (An et al., 2011). The checkerboard assays were also carried out in triplicate.

\section{Etest}

The in vitro interactions between VAN and UA, UA-lipo, $\beta$-lap or $\beta$-lap-lipo were also evaluated by an epsilometer test (Etest) (Sopirala et al., 2010). The Etest was performed with subinhibitory concentrations of active substances B (UA, UA-lipo, $\beta$-lap and $\beta$-lap-lipo) of $1 / 4$ to $1 / 2$ of the respective MIC. The same clinical isolates used in the checkerboard test were adjusted at an optical density of $0.5 \mathrm{McF}$ arland units. They were then seeded onto plates and the VAN Etest strips applied to inoculate Müeller-Hinton agar plates containing UA, UA-lipo, $\beta$-lap or $\beta$-lap-lipo, as well as plates without active substances (control). The plates were then incubated at $35^{\circ} \mathrm{C}$ for $24 \mathrm{~h}$.
The results were interpreted as follows: indifferent effect if the MIC of VAN changed within a 1-fold dilution in the respective plate; an additive effect for a reduction of 2-fold dilutions; and synergic effect for 3-fold dilutions according to the manufacturer's recommendations (Etest application sheet EAS 021). All the experiments were performed in triplicate.

\section{Statistical analysis}

To compare the data of the in vitro interaction assay, one-way analysis of variance (ANOVA) was applied using the Tukey multiple comparison procedure. The statistical data were considered significant at $\mathrm{p}<0.05$.

\section{RESULTS}

\section{Characterization of liposomes}

$\beta$-lap-lipo and UA-lipo had a pH of 7.6 and 7.5, particle size of $110.4 \pm 0.7 \mathrm{~nm}$ and $121.3 \pm 1.8 \mathrm{~nm}$, PDI of 0.319 and 0.362 , zeta potential of $+22.4 \pm 1.8 \mathrm{mV}$ and $+11.8 \pm 0.5 \mathrm{mV}$, and $\%$ EE of $97.4 \pm 1.1 \%$ and $99.0 \pm 2.7 \%$, respectively.

\section{In vitro antimicrobial activity}

The MIC and MBC values of VAN, UA and UA-lipo against MRSA clinical isolates are shown in Table I. As expected, the MIC values of VAN were between 0.25 and $2 \mu \mathrm{g} / \mathrm{mL}$. The MIC values of UA and UA-lipo ranged from 8 to $32 \mu \mathrm{g} / \mathrm{mL}$ and 4 to $8 \mu \mathrm{g} / \mathrm{mL}$, respectively.

\section{In vitro interaction assays}

\section{Checkerboard method}

FICI values obtained in the interaction assays of VAN with UA, UA-lipo, $\beta$-lap or $\beta$-lap-lipo against MRSA clinical isolates are depicted in Figure 1. With regard to the interaction of VAN with UA, an additive interaction for almost all the MRSA clinical isolates was evident $(\mathrm{FICI}=0.51)$, except for LMB 03, which exhibited synergic interactions $($ FICI $=0.26)$. The UA-lipo/VAN association had a synergic effect on all the MRSA clinical isolates tested (FICI $=0.25-0.30)$. Statistically significant differences between the interactions of UA/VAN and UAlipo/VAN were found.

With regard to the in vitro interaction between VAN and $\beta$-lap, synergism was found and the profile remained unaltered when $\beta$-lap was encapsulated into liposomes $(F I C I=0.26-0.50)$, whereas the predominant FICI for other combinations involving these two compounds was 
TABLE I - Antibacterial activity of vancomycin, usnic acid and liposomes containing usnic acid against methicillin-resistant Staphylococcus aureus clinical isolates

\begin{tabular}{lccc}
\hline MRSA & \multicolumn{3}{c}{ MIC/MBC $(\mu \mathrm{g} / \mathrm{mL})$} \\
\cline { 2 - 4 } Strains & VAN & UA & UA-lipo \\
\hline LMB 01 & $0.5 / 2$ & $31 />128$ & $8 />128$ \\
LMB 02 & $0.5 / 1$ & $31 />128$ & $8 />128$ \\
LMB 03 & $2 / 2$ & $31 / 128$ & $8 />128$ \\
LMB 04 & $0.5 / 1$ & $31 / 128$ & $8 />128$ \\
LMB 06 & $0.5 / 2$ & $8 / 16$ & $4 />128$ \\
LMB 07 & $0.5 / 0.5$ & $31 / 32$ & $4 / 32$ \\
LMB 08 & $0.5 / 0.5$ & $31 / 32$ & $8 / 32$ \\
LMB 11 & $0.5 / 1$ & $31 / 128$ & $8 / 32$ \\
LMB 12 & $0.25 / 0.5$ & $8 / 32$ & $8 />128$ \\
LMB 13 & $0.5 / 0.5$ & $31 / 32$ & $8 / 32$ \\
\hline
\end{tabular}

MIC: Minimum Inhibitory Concentration; MBC: Minimum Bactericidal Concentration; MRSA: methicillin-resistant Staphylococcus aureus; LMB: Laboratório de Microbiologia e Imunologia, CAV-UFPE, Brazil; VAN: vancomycin; UA: usnic acid; UA-lipo: UA-loaded liposomes.

0.5 (Figure 1). The statistical analysis showed significant differences between the interactions of $\beta$-lap/VAN and $\beta$-lap-lipo/VAN.

Moreover, there were no statistically significant differences between the interactions of $\beta$-lap-lipo/VAN and UA-lipo/VAN, suggesting that the encapsulation of drugs into liposomes associated with VAN represents a promising therapy against MRSA.

\section{Etest}

The results of the interactions of VAN with UA, UA-lipo, $\beta$-lap or $\beta$-lap-lipo against MRSA clinical isolates using the Etest are listed in Table II. The data obtained using the Etest confirmed the results found with the checkerboard method in $82.5 \%$ of the analysis. Only seven of the forty combinations were not consistent with the other method (17.5\%). The discordant combinations were VAN/ 1 12 MIC UA-lipo for LMB 08; VAN/ 1/4 MIC UA-lipo for LMB 01 and LMB 08; VAN/ $1 / 2$ and $1 / 4$ MIC $\beta$-lap for LMB 08, as well as VAN/ $1 / 4$ MIC $\beta$-lap-lipo for LMB 01 and LMB 06.

\section{DISCUSSION}

The liposomal formulations exhibited suitable physicochemical characteristics, such as particle size, $\mathrm{PDI}$, zeta potential and $\mathrm{pH}$, for future in vivo therapeutic applications (Pinto-Alphandary, Andremont, Couvreur, 2000). Since the administration route of these nanocarriers is intravenous, formulations must have a $\mathrm{pH}$ within the range of neutrality, particle size of below $200 \mathrm{~nm}$ and PDI below 1 to prevent harmful effects on patients, such as acid-base disorders or vessel obstruction (Hinrichs et al., 2006; Elsabahy, Wooley, 2012). In addition, the positive surface charge of the liposomes is important for their electrostatic attraction with the negative cell wall of bacteria (Xia, Kohler, Peschel, 2010). Finally, the high encapsulation rate of AU and $\beta$-lap in liposomes demonstrated the efficiency of the encapsulation method used in this study.

Regarding the susceptibility of MRSA clinical isolates to VAN, the CLSI (2014) defines susceptible strains as having MICs $\leq 2 \mu \mathrm{g} / \mathrm{mL}$, intermediate as MICs $4-8 \mu \mathrm{g} / \mathrm{mL}$ and resistant as MICs $\geq 16 \mu \mathrm{g} / \mathrm{mL}$. The MRSA clinical isolates tested in the present study showed in vitro VAN susceptibility (MICs $\leq 2 \mu \mathrm{g} / \mathrm{mL}$ ). A low efficacy of VAN against MRSA clinical isolates at relatively high in vitro $\mathrm{MIC}_{\mathrm{VAN}}$ values $(1-2 \mu \mathrm{g} / \mathrm{mL})$ is correlated with VAN treatment failures and mortality of patients with MRSA infections. In these cases, novel approaches, as well as combination therapy, are suitable for the treatment of MRSA (Moise et al., 2007; Lodise et al., 2008; Casapao et al., 2015).

The MIC values of UA against MRSA were consistent with data on UA antimicrobial activity previously reported in the literature. In 1995, Lauterwein et al. found usnic acid MICs in the 8 to $16 \mu \mathrm{g} / \mathrm{mL}$ range against MRSA clinical isolates.

UA-lipo had heightened bacteriostatic activity when compared with pure UA, showing a decrease in MIC from 8-32 $\mu \mathrm{g} / \mathrm{mL}$ to $4-8 \mu \mathrm{g} / \mathrm{mL}$. One explanation for the improvement in antimicrobial activity produced by the encapsulation of UA into liposomes is the possible fusion of the nanostructured systems with the cell wall of $S$. aureus, which allows an increased amount of active substance within the bacterial cells. In fact, UA is able to cross biological membranes by modulating its solubility through protonation/deprotonation of $3-\mathrm{OH}$ in a physiological pH environment (Sharma, Jannke, 1996). As recently reported for in silico simulation studies, UA, in its usnate ionic form, causes perturbation in phospholipid bilayers, which undergo a lamellar to nonlamellar transition phase (Nadvorny, Da Silva, Lins, 2014).

In the interaction study by the checkerboard method, the encapsulation of UA into liposomes appeared to be a promoter factor for synergism. The combination therapy of VAN with other antibiotics has clearly emerged as a potential approach to overcome the decrease in bacterial 

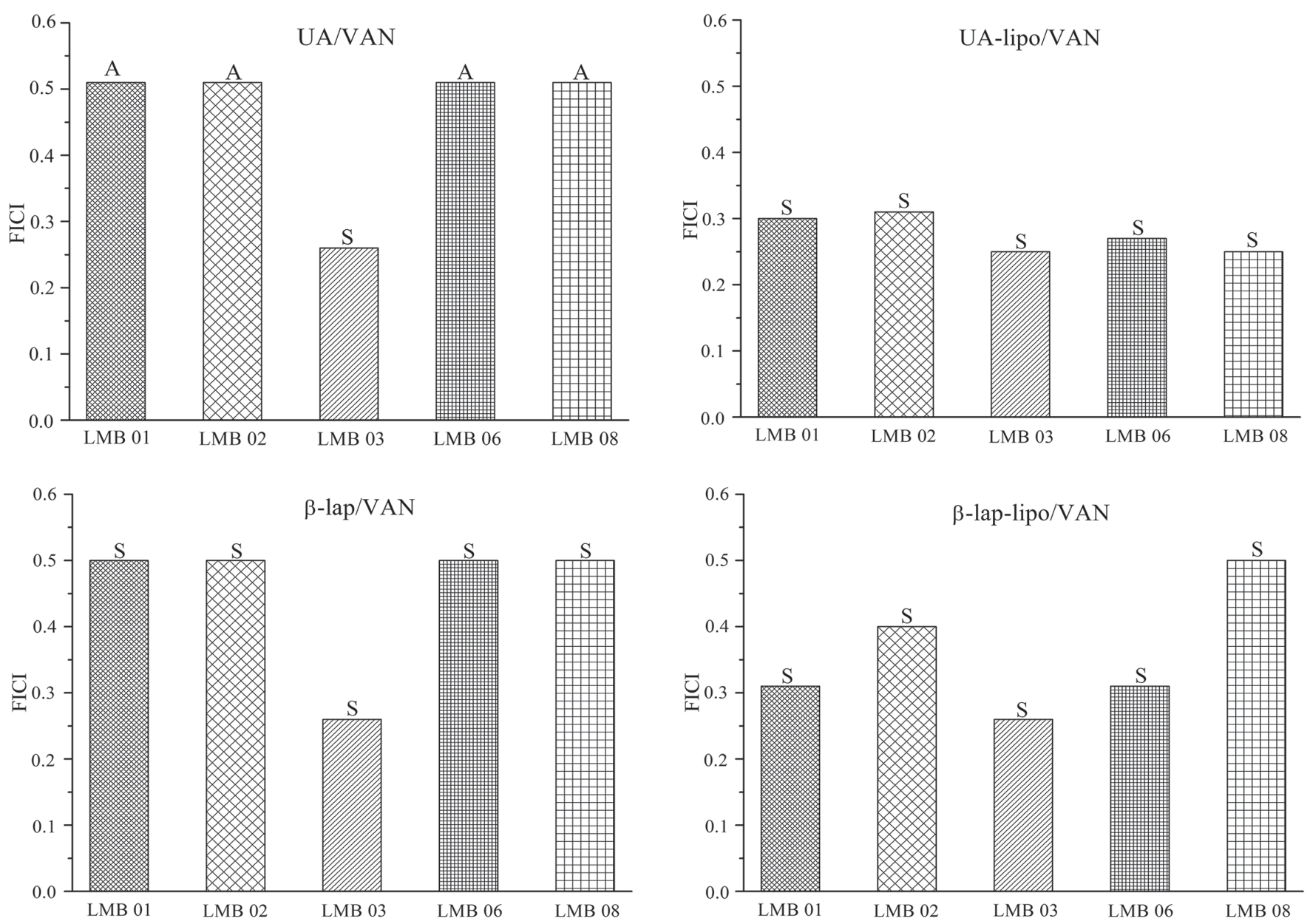

FIGURE 1 - In vitro interactions between VAN and UA, UA-lipo, $\beta$-lap or $\beta$-lap-lipo against MRSA clinical isolates using checkerboard method. FICI: Fractional Inhibitory Concentration Index; LMB: Laboratório de Microbiologia e Imunologia; VAN: vancomycin; UA: usnic acid; $\beta$-lap: $\beta$-lapachone; UA-lipo: UA-loaded liposomes; $\beta$-lap-lipo: $\beta$-lap-loaded liposomes; A: additive interaction; S: synergistic interaction.

TABLE II - In vitro interactions between VAN and UA, UA-lipo, $\beta$-lap or $\beta$-lap-lipo using Etest

\begin{tabular}{|c|c|c|c|c|c|c|c|c|c|c|c|c|c|c|c|c|c|}
\hline \multirow{3}{*}{ MRSA strains } & \multicolumn{17}{|c|}{$\mathrm{MIC}_{\mathrm{VAN}}(\mu \mathrm{g} / \mathrm{mL})$} \\
\hline & \multirow[t]{2}{*}{ VAN } & \multicolumn{4}{|c|}{ VAN/UA } & \multicolumn{4}{|c|}{ VAN/UA-lipo } & \multicolumn{4}{|c|}{ VAN/ $/$-lap } & \multicolumn{4}{|c|}{ VAN/ $\beta$-lap-lipo } \\
\hline & & $\begin{array}{c}1 / 2 \times \mathrm{MIC} \\
\mathrm{UA}\end{array}$ & Int & $\begin{array}{c}1 / 4 \times \mathrm{MIC} \\
\mathrm{UA}\end{array}$ & Int & $\begin{array}{l}1 / 2 \times \text { MIC } \\
\text { UA-lipo }\end{array}$ & Int & $\begin{array}{l}1 / 4 \times \text { MIC } \\
\text { UA-lipo }\end{array}$ & Int & $\begin{array}{c}1 / 2 \times \text { MIC } \\
\beta \text {-lap }\end{array}$ & Int & $\begin{array}{c}1 / 4 \times \text { MIC } \\
\beta \text {-lap }\end{array}$ & Int & $\begin{array}{l}1 / 2 \times \text { MIC } \\
\beta \text {-lap-lipo }\end{array}$ & Int & $\begin{array}{l}/ 4 \times \text { MIC } \\
\beta \text {-lap-lipo }\end{array}$ & Int \\
\hline$\overline{\text { LMB } 01}$ & 0.5 & 0.12 & $\mathrm{~A}$ & 0.12 & $\mathrm{~A}$ & 0.06 & $\mathrm{~S}$ & 0.12 & $\mathrm{~A}$ & 0.06 & $\mathrm{~S}$ & 0.06 & $\mathrm{~S}$ & 0.06 & $\mathrm{~S}$ & 0.12 & $\mathrm{~A}$ \\
\hline LMB 02 & 0.5 & 0.12 & $\mathrm{~A}$ & 0.12 & A & 0.06 & S & 0.06 & $\mathrm{~S}$ & 0.06 & $\mathrm{~S}$ & 0.06 & $\mathrm{~S}$ & 0.06 & $\mathrm{~S}$ & 0.06 & S \\
\hline LMB 03 & 1 & 0.12 & $\mathrm{~S}$ & 0.12 & $\mathrm{~S}$ & 0.12 & S & 0.12 & $\mathrm{~S}$ & 0.12 & $\mathrm{~S}$ & 0.12 & $\mathrm{~S}$ & 0.12 & $\mathrm{~S}$ & 0.12 & $\mathrm{~S}$ \\
\hline LMB 08 & 1 & 0.25 & A & 0.25 & A & 0.25 & A & 0.25 & A & 0.25 & A & 0.25 & A & 0.12 & $\mathrm{~S}$ & 0.12 & S \\
\hline
\end{tabular}

Etest: epsilometer test; MIC: Minimum inhibitory concentration; LMB: Laboratório de Microbiologia e Imunologia (CAV-UFPE, Brazil); Int: in vitro interaction; VAN: vancomycin; UA: Usnic acid; UA-lipo: UA-loaded liposomes; $\beta$-lap: $\beta$-lapachone; $\beta$-lap-lipo: $\beta$-lap-loaded liposomes. A: additive; S: synergistic.

susceptibility to this reference drug. Our findings thus suggest UA, especially encapsulated into liposomes, as a possible promoter to improve VAN antimicrobial activity and proved more significant than results obtained by Segatore et al. (2012). The cited authors analyzed the interaction of UA with five antibiotics used in clinical therapy (clindamycin, erythromycin, gentamicin, oxacillin and levofloxacin) against four MRSA clinical isolates. 
For all isolates studied, the interaction of UA with erythromycin, oxacillin and levofloxacin was indifferent; for clindamycin, the interaction with UA was synergic for only one isolate, yet indifferent for the other three. Synergic interactions of UA with gentamicin were found for two of the four strains and indifferent interaction for the other two.

The results for $\beta$-lap interactions with VAN are in agreement with those of Macedo et al. (2013) for interactions between $\beta$-lap and ampicillin, amoxicillin/ clavulanic acid, cefoxitin, ciprofloxacin or meropenem against five MRSA isolates. A synergism in almost all interactions between $\beta$-lap and antibiotics tested (FICIs = $0.346,0.166,0.375,0.384$ and 0.224 , respectively) was observed, except for the additive interaction between $\beta$-lap and ciprofloxacin against one isolate $(\mathrm{FICI}=0.56)$.

In the past few decades, in vitro synergistic interactions of VAN with antimicrobial agents for antibacterial therapy have been reported. You et al. (2000) investigated the existence of synergism between arbekacin and VAN and compared it with the in vitro interaction of the classical combination of gentamicin and VAN against MRSA isolates resistant to gentamicin. The arbekacin/VAN combination exhibited a synergic effect against twelve of the thirteen isolates tested, whereas the gentamicin/VAN combination was synergistic for only seven.

Some of the combinations assessed in our study (UA-lipo/VAN, $\beta$-lap/VAN and $\beta$-lap-lipo/VAN) seem to be more synergistic than the standard combination (gentamicin/VAN), as the combinations were synergistic in $100 \%$ of the MRSA isolates tested. In 2010, Mahboubi and Bidgoli also evaluated the in vitro interaction of vancomycin with natural products, such as Zataria multiflora essential oil, against MRSA clinical isolates. Similar to our results, the interaction was synergistic between these substances (FICI $=0.32$ ).

The level of agreement between the data from the Etest and the checkerboard methods was notable $(82.5 \%)$ and comparable with previous studies (White et al., 1996; Sopirala et al., 2010). White et al. (1996) investigated the interaction between antibiotics against gram positive and gram negative bacteria using the Etest and checkerboard methods and observed a greater discrepancy of approximately $25 \%$. Sopirala et al. (2010) analyzed the interaction between antibiotics against PanDrug-Resistant Acinetobacter baumannii and found a discrepancy of around $15 \%$ between the results obtained from the two tests in question.

Thus, the results observed in the checkerboard method corroborate the Etest observation performed in our study and these microbial experiments suggested that VAN combined with UA-lipo, $\beta$-lap or $\beta$-lap-lipo synergistically enhanced its antibacterial activity against methicillinresistant Staphylococcus aureus clinical isolates.

\section{CONCLUSION}

Vancomycin exhibited synergism with UA and $\beta$-lap encapsulated into liposomal formulations. Therefore, these natural compounds encapsulated into the drug delivery system may be a potential therapeutic approach for improving the antimicrobial activity of vancomycin in the treatment of MRSA. Further studies in animal models are needed to evaluate the in vivo antimicrobial effect of these combinations and their potential use in the treatment of infections caused by MRSA.

\section{ACKNOWLEDGEMENT}

The authors wish to thank the Brazilian National Research Council (CNPq) for grants \#484574/2011-6. T.G.C. Menezes also thanks the Brazilian Education Ministry (CAPES) for an MSc scholarship.

\section{CONFLICT OF INTEREST}

No conflict of interest is declared.

\section{REFERENCES}

Abed N, Couvreur P. Nanocarriers for antibiotics: A promising solution to treat intracellular bacterial infections. Int $\mathrm{J}$ Antimicrob Agents. 2014;43(6):485-496.

An J, Zuo GY, Hao XY, Wang GC, Li ZS. Antibacterial and synergy of a flavonol rhamnoside with antibiotics against clinical isolates of methicillin-resistant Staphylococcus aureus (MRSA). Phytomedicine. 2011;18(11):990-993.

Casapao AM, Lodise TP, Davis SL, Claeys KC, Kullar R, Levine DP, et al. Association between vancomycin day 1 exposure profile and outcomes among patients with Methicillin-Resistant Staphylococcus aureus infective endocarditis. Antimicrob Agents Chemother. 2015;59(6):2978-2985.

Cavalcanti IMF, Mendonça EAM, Lira MCB, Honrato SB, Camara CA, Amorim RVS, et al. The encapsulation of $\beta$-lapachone in 2-hydroxypropyl- $\beta$-cyclodextrin inclusion complex into liposomes: A physicochemical evaluation and molecular modeling approach. Eur J Pharm Sci. 2011;44(3):33240. 
Cavalcanti IMF, Pontes-Neto JG, Kocerginsky PO, BezerraNeto AM, LIMA JLC, Lira-Nogueira MCB, et al. Antimicrobial activity of $\beta$-lapachone encapsulated into liposomes against meticillin-resistant Staphylococcus aureus and Cryptococcus neoformans clinical strains. J Glob Antimicrob Resist. 2015;3(2):103-108.

Clinical and Laboratory Standards Institute. CLSI. Performance standards for antimicrobial susceptibility testing; twenty-fourth informational supplement (M100-S24). Wayne, USA: CLSI; 2014.

Drulis-Kawa Z, Dorotkiewicz-Jach A. Liposomes as delivery systems for antibiotics. Int J Pharm. 2010;387(1-2):187-198.

Elsabahy M, Wooley K.L. Design of polymeric nanoparticles for biomedical delivery applications. Chem Soc Rev. 2012;41(7):2545-2561.

Han D, Matsumaru K, Rettori D, Kaplowitz N. Usnic acid induced necrosis of culture mouse hepatocytes: inhibition of mitochondrial function and oxidative stress. Biochem Pharmacol. 2004;67(3):439-451.

Hinrichs WLJ, Manceñido FA, Sanders NN, Braeckmans K, De Smedt SC, Demeester J, Frijlink HW. The choice of a suitable oligosaccharide to prevent aggregation of PEGylated nanoparticles during freeze thawing and freeze drying. Int $\mathrm{J}$ Pharm. 2006;311(1-2):237-244.

Huh AJ, Kwon YJ. "Nanoantibiotics": A new paradigm for treating infectious diseases using nanomaterials in the antibiotics resistant era. J Control Release. 2011;156(2):128-145.

Jackson C, Agboke A, Nwoke V. In vitro evaluation of antimicrobial activity of combinations of nystatin and Euphorbia hirta leaf extract against Candida albicans by the checkerboard method. J Med Plants Res. 2009;3(9):666-669.

Kristmundsdoóttir T, Aradójattir HA, Ingólfsdóttir K, Ogmundsdóttir HM. Solubilization of the lichen metabolite (?)-usnic acid for testing in tissue culture. J Pharm Pharmacol. 2002;54(11):1447-1452.

Lauterwein M, Oethinger M, Belsner K, Peters T, Marre R. In vitro activities of the lichen secondary metabolites vulpinic acid, (+)-usnic acid, and (-)-usnic acid against aerobic and anaerobic microorganisms. Antimicrob Agents Chemother. 1995;39(11):2541-2543.
Lira MCB, Siqueira-Moura MP, Rolim-Santos HML, Galetti FCS, Simioni AR, Santos NP, et al. In vitro uptake and antimycobacterial activity of liposomal usnic acid formulation. J Liposome Res. 2009;9(1):49-58.

Lodise TP, Graves J, Evans A, Graffunder E, Helmecke M, Lomaestro BM, et al. Relationship between vancomycin MIC and failure among patients with methicillin-resistant Staphylococcus aureus bacteremia treated with vancomycin. Antimicrob Agents Chemother. 2008;52(9):3315-3320.

Macedo L, Fernandes T, Silveira L, Mesquita A, Franchitti AA, Ximenes EA. $\beta$-lapachone activity in synergy with conventional antimicrobials against methicillin resistant Staphylococcus aureus strains. Phytomedicine. 2013;21(1):25-29.

Mahboubi M, Bidgoli FG. Antistaphylococcal activity of Zataria multiflora essential oil and its synergy with vancomycin. Phytomedicine. 2010;17(7):548-550.

Moise PA, Sakoulas G, Forrest A, Schentag JJ. Vancomycin in vitro bactericidal activity and its relationship to efficacy in clearance of methicillin-resistant Staphylococcus aureus bacteremia. Antimicrob Agents Chemother. 2007;51(7):25822586 .

Nadvorny D, Da Silva JBP, Lins RD. Anionic form of usnic acid promotes lamellar to nonlamellar transition in DPPC and DOPC membranes. J Phys Chem B. 2014;118(14):3881-3886.

Nasongkla N, Wiedmann AF, Bruening A, Beman M, Ray D, Bornmann WG, et al. Enhancement of solubility and bioavailability of $\beta$-lapachone using cyclodextrin inclusion complexes. Pharm Res. 2003;20(10):1626-1633.

Pinto-Alphandary H, Andremont A, Couvreur P. Targeted delivery of antibiotics using liposomes and nanoparticles: research and applications. Int J Antimicrob Agents. 2000;13(13):155-168.

Segatore B, Bellio P, Setacci D, Brisdelli F, Piovano M, Garbarino JÁ, et al. In vitro interaction of usnic acid in combination with antimicrobial agents against methicillinresistant Staphylococcus aureus clinical isolates determined by FICI and $\Delta \mathrm{E}$ model methods. Phytomedicine. 2012;19(34):341-347.

Sharma RK, Jannke PJ. Acidity of usnic acid. Indian J Chem. 1966;4(1):16-18. 
Silva JL, Mesquita ARC, Ximenes EA. In vitro synergic effect of $\beta$-lapachone and isoniazid on the growth of Mycobacterium fortuitum and Mycobacterium smegmatis. Mem Inst Osw Cruz. 2009;104(4):580-582.

Sopirala MM, Mangino JE, Gebreyes WA, Biller B, Bannerman T, Balada-Llasat JM, et al. Synergy testing by Etest, microdilution checkerboard, and time-kill methods for pan-drug-resistant Acinetobacter baumannii. Antimicrob Agents Chemother. 2010;54(11):4678-4683.

Xia G, Kohler T, Peschel A. The wall teichoic acid and lipoteichoic acid polymers of Staphylococcus aureus. Int J Med Microbiol. 2010;300(2-3):148-154.

White RL, Burgess DS, Manduru M, Bosso JA. Comparison of three different in vitro methods of detecting synergy: timekill, checkerboard, and E test. Antimicrob Agents Chemother. 1996;40(8):1914-1918.
Worthington RJ, Melander C. Combination approaches to combat multidrug-resistant bacteria. Trends Biotech. 2013;31(3):177-184.

You I, Kariyama R, Zervos MJ, Kumon H, Chow JW. In vitro activity of arbekacin alone and in combination with vancomycin against gentamicin- and methicillin-resistant Staphylococcus aureus. Diagn Microbiol Infect Dis. 2000;36(1):37-41.

Zecconi A, Scali F. Staphylococcus aureus virulence factors in evasion from innate immune defenses in human and animal diseases. Immunol Letters. 2013;150(1-2):12-22.

Received for publication on $17^{\text {th }}$ November 2016 Accepted for publication on $18^{\text {th }}$ October 2017 